\section{P-110 IMPLEMENTING REHABILITATIVE PALLIATIVE CARE - A TEAM APPROACH}

Nicola Silk. Douglas Macmillan Hospice, Stoke on Trent, UK

10.1136/bmjspcare-2016-001245.133

Background Following the publication of Hospice UK's rehabilitative palliative care document (2015), it was highlighted that the hospice could challenge its current practice and welcome change with regards to rehabilitative palliative care. Whilst providing holistic, person-centred specialist services it was identified that the hospice could better tailor services for patients who were appropriate for this level of intervention.

Aim Develop the "Improving Care group in Rehabilitative Palliative Care" to identify and implement aspects of rehabilitative palliative care in and around the hospice.

Method A team of multidisciplinary staff from the in-patient unit, day therapy unit and the community led by the physiotherapist and including nursing staff, social work, allied health professionals and medics used the rehabilitative palliative care document and checklist to highlight all areas of concern or where further work would be beneficial and prioritise their actions accordingly

Result Monthly meetings were held and focus groups on separate topics were created.

Communication - Including handovers, leaflets for patients and relative, information boards, admission and discharge documentation

Goal Setting - Patient-focused goal setting documentation to be created, incorporated into weekly MDT and adapted in line with requirements

Rehabilitation care plans - care plan development with a focus on enabling patients facilitating independence and self-management

Environment - ensuring all hospice areas are enabling and facilitate independence and normality.

Education - Sharing information, knowledge and skills to support staff to change in practice.

Activities - Offer activities encouraging patients to access communal areas and interacting in activities

Admissions/Discharges - Ensuring information sharing with regards to admission and discharging patients is accurate and effective to ensure all aspects of care are appropriate

Conclusion Working as a team, the hospice staff have been able to successfully identify areas for change and work effectively to implement aspects of rehabilitative palliative care. This work is ongoing to make a difference to patients in our care.

\section{P-111 THE IMPORTANCE OF A DEDICATED FORWARD THINKING HOUSEKEEPING TEAM WORKING IN A CLINICAL ENVIRONMENT}

Angela Marsh, Paul Munyard. Douglas Macmillan Hospice, Stoke on Trent, UK

10.1136/bmjspcare-2016-001245.134

The housekeeping team worked daily to ensure the cleaning and standards of the hospice where completed to a high standard but what standard? The cleaning team didn't have a choice on decision making or cleaning standards so we introduced a team leader who recognised the need for change to have a proactive approach to cleanliness, decontamination and infection prevention. We implemented change and for the first time the Housekeeping team leader sat on the infection prevention and cleanliness committee (IPCC) not only being an attendee but an active member to bring best practice and ideas forward. New policies and procedures where written, housekeeping staff re-trained and mentored. One positive improvement was to introduce Green "I am clean" stickers. These where easily recognisable by all; nursing staff, patients and families were confident knowing that the rooms where clean and the housekeeping team found this a positive communication skill.

We then needed to review that change to ensure this was an improvement. So we created cleanliness and operational reports approved by our IPCC. We based this on the PLACE model and tailored the areas of inspection to Cleanliness and Operation of patients' areas to ensure these are fit for use as well as clean. The report has 30 domains to score against which gives an overall rating of:

\begin{tabular}{ll}
\hline Green & Control is sufficient \\
Amber & Further control needed for improvement \\
Red & Considerable need for improvement \\
\hline
\end{tabular}

Once the report is complete, findings are reported to IPCC. In future it's hoped we can start to extract data which then can be used further to support training and best practice sharing. This forward thinking will certainly bring the housekeeping team to the forefront of the hospice rather than feeling undervalued and proves that collaborative working on this domain has been highly positive move.

\section{P-112 IS THE MANAGEMENT OF HEALTHCARE-ASSOCIATED INFECTIONS IN HOSPICES A MISNOMER?}

Lynda Price. Helen and Douglas House, Oxford, UK

\subsection{6/bmjspcare-2016-001245.135}

There is increasing evidence, albeit hospital based, that healthcare-associated infections (HCAIs) are amongst the leading communicable and preventable cause of morbidity and mortality (Tulchinsky and Varavikova, 2009, NICE, 2011). With the multifaceted delivery of healthcare, the risk of infection increases (Apisarnthanarak et al., 2012). Hospices are unique settings (with extremely vulnerable patient populations), but operate within wider healthcare contexts - so what is their role in managing HCAIs?

The aim of this study, using a critical literature review, was to identify and discuss the current knowledge available about the management of HCAIs in hospices. The study was triggered by a number of incidents where patients were due to be admitted to the hospice as part of a stepped discharge from hospital. In each case the patient was being treated for infections with multi-drug resistant organisms (MDROs), but their infection status was not handed over. Preliminary discussions with infection prevention and control practitioners in other hospices suggested inconsistencies in the management of the HCAI risk.

Five key themes emerged from the study - the availability of current empirical knowledge, risks associated with HCAIs, the impact HCAIs and of infection prevention and control strategies on the quality of life for patients in palliative care, the ethos of palliative care, and screening. 
The review found little empirical evidence that HCAIs are managed in hospices, and concluded that the management of HCAIs in hospices is a misnomer. The broad purpose of this study was not to judge infection prevention and control practices in hospices, but to trigger thought and debate, and to develop collaborative approaches to HCAIs.

The study recommends further qualitative research based on patient perceptions, on the impact of HCAIs, and on infection prevention and control strategies. This knowledge could be used to inform strategies and interventions for palliative care patients.

\section{P-113 SERVICE INNOVATION TO MEET THE INCREASING DEMAND FOR DIETETICS IN PALLIATIVE CARE}

Anne Holdoway, Kathryn King, Ruth Gretton. Dorothy House Hospice Care, Bradford on Avon, UK

\subsection{6/bmjspcare-2016-001245.136}

Background Many life-limiting conditions negatively impact on an individual's ability to eat and drink pre-disposing an individual to malnutrition including its adverse consequences. Enjoyment and pleasure from eating and drinking can decline and diet becomes a source of psychological stress and anxiety. Although there exists a sound clinical rationale for dietetic interventions to optimise nutrition and alleviate symptoms dietetic services to hospices remains limited. In our own setting a specialist dietetic post (0.23 w.t.e.) was established in 2012. The service evolved to offer:

- a nutrition component to the patient rehabilitation and carer courses

- one to one consultations for patients with complex conditions requiring specialist dietary advice

- education and training for staff (internal and external).

Demand quickly out-stripped provision and therefore a creative, cost-effective solution was sought to continue to support patients and carers.

Aim To meet growing demand for dietetics with a newly created dietetic assistant post.

Results Following scoping and recruitment to the post, training was provided through case studies, supervision and e-learning. Since recruitment it has been possible to

- Further develop resources including a range of 'Handy Hints' (printed and web-based)

- Provide timely first-line dietetic interventions for patients and carers.

- Better support the specialist nurses.

- Streamline the use of oral nutritional supplements saving costs for the CCG.

A tool to identify patients requiring referral to the specialist dietitian for complex management has been validated and introduced.

Patient feedback in the form of patient evaluation and outcome measures have demonstrated the positive impact of the new service.

Conclusion To our knowledge this is the first hospice-based dietetic assistant post in the UK. Supervised by the specialist dietitian, this post has been better able to meet patient's needs. Similar posts across the UK would assist in meeting the growing demands of clients requiring supportive care who would benefit from diet modifications to maximise quality of life.

\section{P-114 A HOLISTIC APPROACH TO NUTRITION AND DIET IN PALLIATIVE CARE}

Jill Souter, Agata Czerwinska, Sarah Cooley, Anne Holdoway. Dorothy House Hospice Care, Bradford on Avon, UK

10.1136/bmjspcare-2016-001245.137

Background In response to the Care Quality Commission and NHS contract requirements, a validated screening tool; the Malnutrition Universal Screening Tool 'MUST' was introduced into our hospice setting in 2012. Subsequent research amongst nursing staff confirmed lack of confidence in 'MUST' in identifying and guiding decisions for appropriate nutritional care. A project team was established to investigate how we might achieve appropriate nutritional care taking into account the changing and complex needs of patients and carers in the hospice setting.

Aims To identify nutritional issues experienced and develop a nutrition assessment tool to sensitively determine and deliver nutritional care to meet the needs of patients and carers.

Methods A practice development approach was utilised engaging all stakeholders. The approach included:

- questionnaires to staff

- discussion forums involving members of the healthcare team, patients, carers and catering.

- qualitative interviews with patients/carers.

Findings were used to initiate change and refine and validate a nutrition assessment tool.

Results Results from the questionnaires and forums identified issues affecting the delivery of optimal nutritional care. Solutions were identified and tested, patient and carer participation was a key component. A new approach to menu planning, including 13-day cycles, menu choices and portion size was developed. A new nutrition assessment tool - the 'Patient Led Assessment for Nutritional Care' (PLANC) was developed, tested, refined and validated to identify nutritional issues and determine care taking into account the stage of disease. Nutrition and hydration were integrated into the shared patient record. New pathways and resources were created to address common nutritional problems with triggers for escalation management to a specialist dietitian and dietetic assistant when required.

Conclusion The practice development process for change fitted well with the ethos of the organisation and resulted in practice improvements to enhance care. The 'PLANC' tool for nutrition assessment has been integrated into care across the settings.

\section{P-115 A HOSPICE BREATHLESSNESS MANAGEMENT INTERVENTION SERVICE - A BLEND OF THE OLD WITH THE NEW!}

${ }^{1,2}$ Declan Cawley, ${ }^{1}$ Clare Horne. ${ }^{1}$ Pilgrims Hospices, Canterbury, UK; ${ }^{2}$ University Of Kent

\subsection{6/bmjspcare-2016-001245.138}

Background Breathlessness is one of the most distressing symptoms reported in the literature with the prevalence rising with the vast multi-morbidity people are living with. The breathlessness management intervention for patients with a variety of mixed pathologies but they have the commonality of refractory, end stage breathlessness. The approach employs non-pharmacological approaches but clearly can be in tandem to pharmacological agents. 